\title{
Comparação do estado nutricional infantil com utilização de diferentes curvas de crescimento ${ }^{1}$
}

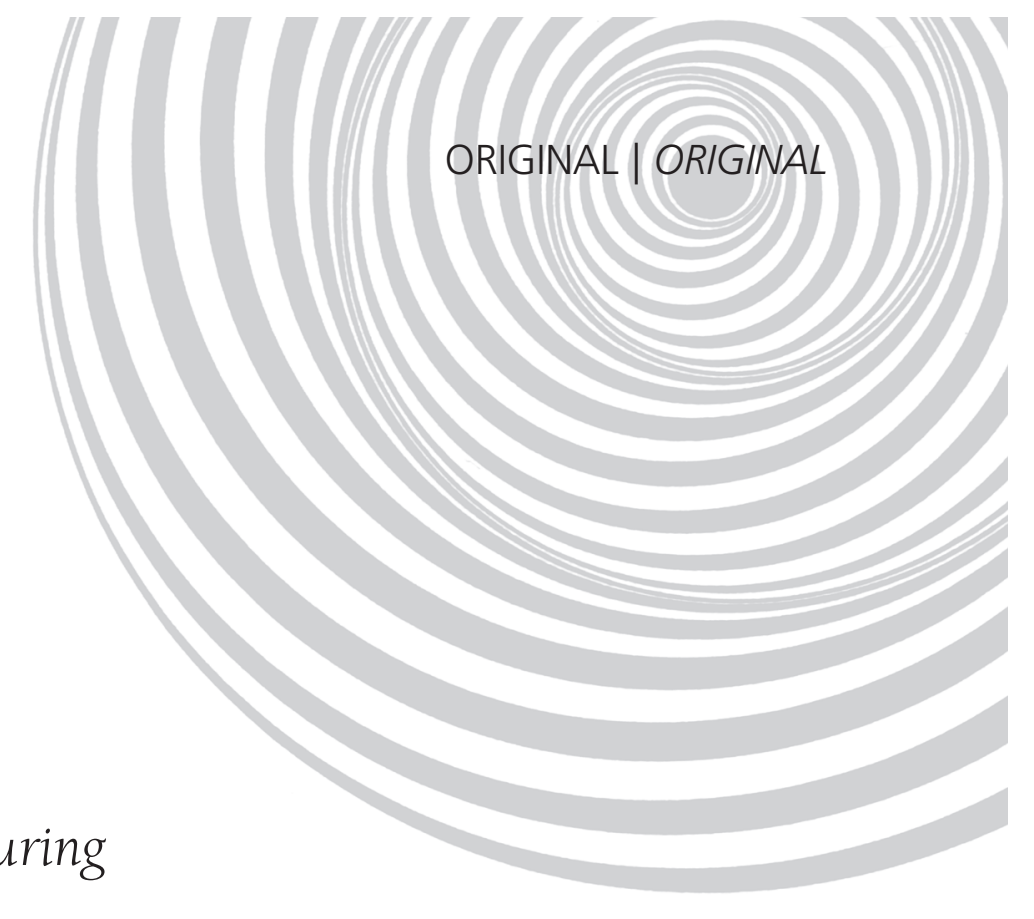

\section{Comparison of the nutritional status during childhood with different growth curves}

\author{
Naiara SPERANDIO 2 \\ Luciana Ferreira da Rocha SANT'ANA² \\ Sylvia do Carmo Castro FRANCESCHINI ${ }^{2}$ \\ Silvia Eloiza PRIORE²
}

RES U M O

\section{Objetivo}

Comparar a classificação do estado nutricional de pré-escolares segundo três curvas de crescimento.

\section{Métodos}

Estudo transversal realizado com 350 crianças de 2 a 5 anos de instituições públicas e particulares do município de Viçosa (MG). A partir do peso e da estatura foram obtidos os índices antropométricos: peso para idade, peso para estatura, índice de massa corporal para idade e estatura para idade. Para classificação de peso insuficiente (menor que - 1 escore-Z), eutrofia e excesso de peso (maior que +1 escore-Z), foram consideradas as curvas do National Center for Health Statistics 1977, Centers for Disease Control 2000 e World Health Organization 2006. Para avaliar a concordância entre as curvas, foi utilizada a estatística Kappa. Consideraram-se o National Center for Health Statistics, já recomendado pelo Ministério da Saúde, e a World Health Organization, atual recomendação, como padrão de referência, a fim de se compararem os valores de sensibilidade, especificidade, valor preditivo positivo e valor preditivo negativo.

\section{Resultados}

A prevalência de peso insuficiente foi maior segundo o Centers for Disease Control. Já com relação ao excesso de peso e deficit estatural, maiores prevalências foram obtidas pela World Health Organization. O National Center for Health Statistics e a World Health Organization apresentaram valores mais próximos de sensibilidade e valor preditivo positivo quando comparados com Centers for Disease Control, assim como também apresentaram melhor correlação $(k>0,61)$.

\footnotetext{
1 Artigo elaborado a partir projeto de iniciação científica intitulado "Estimativa da prevalência de inadequação de nutrientes e de anemia em crianças do município de Viçosa (MG)". Projeto este derivado de um estudo multicêntrico intitulado "Nutri Brasil infância". Apoio: Conselho Nacional de Desenvolvimento Científico e Tecnológico, processo n.106823/2008-6.

2 Universidade Federal de Viçosa, Curso de Pós-Graduação Stricto Sensu e Ciências da Nutrição. Av. P.H. Rolfs, s/n., Campus Universitário, 36570-000, Viçosa, MG, Brasil. Correspondência para/Correspondence to: N. SPERANDIO. E-mail: $<$ naiarasperandio@yahoo.com.br>.
} 
566 | N. SPERANDIO et al.

\section{Conclusão}

Foram encontradas diferenças nas prevalências de deficits nutricionais, tendo as curvas da World Health Organization apresentado maiores valores de sensibilidade. A adoção dessas curvas pelos profissionais da área da saúde permite uma avaliação mais adequada do crescimento infantil por tratar-se de um padrão de crescimento atual, recomendado para uso internacional.

Termos de indexação: Antropometria. Avaliação nutricional. Estado nutricional. Pré-escolar.

\section{A B S T R A C T}

\section{Objective}

This study compared the classification of the nutritional status of preschoolers according to three different growth curves.

\section{Methods}

This cross-sectional study included 350 children aged 2 to 5 years from public and private institutions in the county of Viçosa (MG). The following anthropometric indices were obtained from weight and height measurements: weight-for-age, weight-for-height, body mass index-for-age and height-for-age. The classification of underweight (below -1Z-score), well nourished and overweight (above $+1 Z$-score) was done according to National Center for Health Statistics 1977, Centers for Disease Control and Prevention 2000 and World Health Organization 2006 curves. Kappa statistics was used to evaluate conformity among curves. The National Center for Health Statistics was considered, which is already recommended by the Ministry of Health, and the World Health Organization, which is currently recommended as the reference standard, to compare the sensitivity and specificity values, positive predictive value and negative predictive value.

\section{Results}

The prevalence of underweight was higher when the Centers for Disease Control and Prevention curves were used. On the other hand, regarding overweight and stunting, the World Health Organization curves resulted in the highest prevalence. Both the World Health Organization and the National Center for Health Statistics curves showed closest sensitivity and positive predictive values, as well as better correlation $(k>0.61)$, when compared with the Centers for Disease Control and Prevention curves.

\section{Conclusion}

There are differences in the prevalence of nutritional deficits, and the World Health Organization curves were the ones which showed the highest sensitivities. The use of these curves by health professionals enables a more adequate evaluation of growth during childhood, since it regards a current growing pattern which is also recommended for international use.

Index terms: Anthropometry. Nutrition assessment. Nutritional status. Child, preschool.

\section{N T R O D U Ç Ã O}

O estado nutricional infantil é um indicador de saúde global; assim, monitorar o crescimento e o ganho ponderal permite detectar possíveis agravos à saúde e riscos nutricionais ${ }^{1,2}$. A avaliação das condições de saúde da população possibilita o planejamento de estratégias de intervenção mais eficazes e eficientes ${ }^{3}$.

A antropometria é utilizada principalmente devido ao baixo custo e à facilidade de aferição, sendo as medidas mais utilizadas peso e estatura, e as associações constituem os índices antropométricos ${ }^{4,5}$. Os índices adotados para avaliação do estado nutricional infantil são: peso/idade (P/I), peso/estatura (P/E), estatura/idade (E/I) e Índice de Massa Corporal (IMC)/idade ${ }^{6}$.

Para o diagnóstico nutricional, os resultados obtidos pelos índices devem ser comparados com curvas de crescimento de referência. O referencial do National Center for Health Statistics (NCHS/1977) foi recomendado pela Organização Mundial da Saúde (OMS) e adotado pelo Ministério da Saúde durante muitos anos ${ }^{7}$, contudo 
ele apresentava limitações que permitiam questionamentos referentes a sua adoção, como os fatos de os dados incluírem apenas estudos realizados nos Estados Unidos e de os menores de dois anos terem sido alimentados com fórmulas infantis, contradizendo a recomendação da OMS de aleitamento materno exclusivo até os seis meses de idade ${ }^{8}$.

Surgiu então a necessidade da publicação de um novo referencial que corrigisse as limitações do NCHS/1977. Em 2000, o Center for Disease Control and Prevention (CDC) publicou uma revisão do NCHS/1977 a fim de corrigir algumas de suas falhas. As principais mudanças foram: utilização de técnicas estatísticas mais aprimoradas, aumento do tamanho da amostra, maior diversidade racial e étnica, padronização metodológica, extensão de todas as curvas de crescimento até os 20 anos de idade e preconização do uso do índice de massa corporal para idade (IMC/Idade $)^{9,10}$. Com relação ao aleitamento materno, a referência do CDC/2000 é composta por um conjunto de crianças alimentadas por fórmulas infantis e leite materno, sendo assim o problema atenuado, mas não corrigido ${ }^{10,11}$.

A OMS conduziu um estudo multicêntrico no período de 1997 a 2003, que gerou o atual padrão de crescimento para avaliação do estado nutricional de crianças de zero a cinco anos. Coletaram-se informações de aproximadamente 8500 crianças de diferentes grupos étnicos e culturais ${ }^{11}$. Alguns aspectos importantes desse estudo é sua amostra internacional e multiétnica e o fato de que todas as crianças receberam aleitamento materno exclusivo ou predominante até os quatro meses de idade, colocado como norma ideal para crescimento. Além disso, ele descreve o crescimento infantil normal sob condições ótimas, podendo ser as curvas de crescimento da WHO/2006 utilizadas para avaliar o estado nutricional de crianças de qualquer parte do mundo, independentemente da etnia ou das condições socioeconômicas ${ }^{11,12}$.

Considerando que a utilização de diferentes curvas de crescimento para classificação do estado nutricional infantil cria possibilidades de diferentes resultados, julgou-se necessário analisar comparativamente o estado nutricional de pré-escolares a partir da utilização dos referenciais do NCHS/1977, CDC/2000 e WHO/2006.

\section{MÉ T O D O S}

Trata-se de um estudo transversal, integrante de um projeto multicêntrico que envolve mais oito cidades brasileiras, realizado no município de Viçosa-MG (Caxias do Sul-RS, Manaus-AM, Natal-RN, Recife-PE, Brasília-DF, Cuiabá-MT, Rio de Janeiro-RJ e São Paulo-SP), intitulado "Nutri Brasil Infância", cujo objetivo foi analisar a prevalência de inadequação de nutrientes entre as crianças brasileiras, além da avaliação antropométrica. Participaram do estudo crianças de ambos os sexos, de 2 a 5 anos de idade, de instituições públicas e particulares. Os dados foram coletados durante o ano de 2007 e início de 2008.

O cálculo amostral inicial das crianças em cada cidade baseou-se no objetivo principal do estudo maior. Assim, em cada cidade, avaliaram-se, no mínimo, 250 crianças de creches públicas e 100 de particulares, totalizando 350 crianças, considerando a prevalência de inadequação de nutrientes estimada em $65 \%$, margem de erro de $5 \%$ e intervalo de confiança de 95\% (IC95\%). Para este trabalho, entretanto, considerando seu objetivo e a ausência de estudos nacionais ou regionais semelhantes, encontrou-se poder superior a 90\% para a amostra calculada inicialmente, com a estimativa de diferença de consumo de 10\% para energia e macronutrientes entre as crianças de creches particulares e públicas. A proporção de entrevistas segundo rede de ensino baseou-se no número de matrículas nas creches e pré-escolas registradas no Censo Escolar do Ministério da Educação (2005) para os estados participantes do estudo.

O presente trabalho apresenta os dados das 350 crianças avaliadas no município de Viçosa (MG). Para participar do estudo, as crianças deveriam estar regularmente matriculadas nas insti- 
tuições de ensino e apresentar o Termo de Consentimento Livre e Esclarecido (TCLE) devidamente assinado pelos responsáveis. Foram excluídas crianças enfermas e que não estavam presentes nos dias da coleta dos dados.

As medidas antropométricas foram obtidas segundo as recomendações da $\mathrm{OMS}^{13}$. Para obtenção do peso, utilizou-se balança digital com capacidade máxima de $150 \mathrm{~kg}$ e precisão de $50 \mathrm{~g}$. Somente roupas íntimas foram permitidas durante a pesagem. Para aferição da estatura, as crianças foram medidas em pé, descalças, com auxílio de um antropômetro móvel, a 90 graus em relação ao piso.

A partir das medidas antropométricas (peso e estatura), foram obtidos os índices: peso para idade, peso para estatura, estatura para idade e índice de massa corporal para idade (IMC/l), por meio dos programas Epi Info versão 2002 (CDC, Atlanta, Geórgia, EUA) e WHO Anthro versão Beta (WHO, Departament of Nutrition, Genebra, Suiça). As curvas de crescimento utilizadas foram NCHS/1977, CDC/2000 e WHO/2006.

Para os índices P/I, P/E e IMC/I, consideraram-se valores abaixo de -1 escore- $Z$ como peso insuficiente, eutrofia, entre -1 e +1 escore- $Z$ e excesso de peso acima de +1 escore- $Z$. Segundo o índice E/l, considerou-se como estatura inadequada valores abaixo de -1 escore-Z. A classificação de peso insuficiente corresponde ao agrupamento do risco de baixo peso (entre -2 e -1 escore-Z) e baixo peso ( $<-2$ escore-Z), uma vez que a prevalência de risco de baixo peso encontrada foi baixa. O mesmo foi considerado para estatura inadequada, que corresponde ao risco de baixa estatura (entre -2 e -1 escore-Z) e baixa estatura (<-2 escore-Z).

A análise estatística foi realizada com o auxilio do software Sigma-Stat ${ }^{14}$. Para mensurar a concordância entre as referências antropométricas, utilizou-se a estatística Kappa $(k)^{15}$. Adotou-se o nível de significância estatística, probabilidade inferior a $5 \%(p<0,05)$.

Considerou-se a WHO/2006, atual recomendação do Ministério da Saúde, e o NCHS/1977, antiga recomendação, ambos como padrão de referência para o cálculo de sensibilidade, especificidade, valor preditivo positivo e valor preditivo negativo.

Este trabalho foi aprovado pelo Comitê de Ética da Universidade Federal de Viçosa, sob protocolo $n^{\circ}$ 072/2008.

\section{RES U LT A D OS}

A média de idade foi de 3,8 anos ( $D P=0,95$ anos) e a prevalência de crianças do sexo feminino foi de $49,7 \%$ ( $n=174)$.

Independentemente da curva de crescimento utilizada e do sexo, a prevalência de excesso de peso foi maior que a de peso insuficiente (Tabela 1). A prevalência de peso insuficiente foi maior quando utilizada a proposta do CDC/2000, exceto para o índice $\mathrm{P} / \mathrm{E}$, em que a maior prevalência encontrada foi pelo NCHS/1977.

Em relação ao excesso de peso, a maior prevalência foi pela proposta da WHO/2006, exceto para o sexo feminino, no índice $P / E$, em que a maior prevalência foi pelo NCHS/1977.

A prevalência de estatura inadequada foi maior quando adotada a WHO/2006. Destaca-se a diferença entre os sexos, tendo sido encontrada maior prevalência de estatura inadequada no sexo feminino, independente da curva de crescimento utilizada (Tabela 2).

A concordância entre os referenciais antropométricos, avaliada pela estatística Kappa, apresentou valores de 0,16 a 0,91, tendo sido considerados como boa concordância valores de kappa superiores a 0,61 , como colocado por Landis \& Koch $^{16}$ (Tabela 3).

A concordância mais fraca foi entre as propostas do CDC com NCHS e do CDC com WHO, para peso inadequado do sexo feminino, segundo índice P/I. O CDC não apresentou boa concordância para classificação de peso inadequado com as demais curvas; já o NCHS e a WHO apresentaram boa concordância $(k>0,61$, para classificação do estado nutricional em ambos os sexos. 
Tabela 1. Estado nutricional infantil pelos índices P/I, P/E e IMC/I, segundo as referências antropométricas e sexo de pré-escolares do município de Viçosa (MG), 2008.

\begin{tabular}{|c|c|c|c|c|c|c|c|}
\hline \multirow{3}{*}{ Índices } & \multirow{3}{*}{ Sexo/Referências } & \multicolumn{6}{|c|}{ Estado nutricional } \\
\hline & & \multicolumn{2}{|c|}{ Peso insuficiente } & \multicolumn{2}{|c|}{ Eutrofia } & \multicolumn{2}{|c|}{ Excesso de peso } \\
\hline & & $\mathrm{n}$ & $\%$ & $n$ & $\%$ & $\mathrm{n}$ & $\%$ \\
\hline & Meninos $(n=176)$ & & & & & & \\
\hline \multirow[t]{3}{*}{$P / l$} & $\mathrm{NCHS}$ & 16 & 9,0 & 134 & 76,1 & 26 & 14,9 \\
\hline & CDC & 21 & 12,0 & 111 & 63,0 & 44 & 25,0 \\
\hline & WHO & 13 & 7,4 & 115 & 65,3 & 48 & 27,3 \\
\hline \multirow[t]{3}{*}{ P/E } & NCHS & 26 & 14,8 & 106 & 60,2 & 44 & 25,0 \\
\hline & CDC & 24 & 2,3 & 120 & 79,5 & 32 & 18,2 \\
\hline & WHO & 17 & 9,6 & 110 & 62,5 & 49 & 27,9 \\
\hline \multirow[t]{3}{*}{ IMC/I } & $C D C$ & 26 & 14,8 & 115 & 65,3 & 35 & 19,9 \\
\hline & WHO & 13 & 7,4 & 117 & 66,5 & 46 & 25,1 \\
\hline & Meninas $(n=174)$ & & & & & & \\
\hline \multirow[t]{3}{*}{ P/l } & $\mathrm{NCHS}$ & 13 & 7,5 & 125 & 71,8 & 36 & 20,7 \\
\hline & CDC & 25 & 14,4 & 107 & 61,5 & 42 & 24,1 \\
\hline & WHO & 14 & 8,0 & 116 & 66,7 & 44 & 25,3 \\
\hline \multirow[t]{3}{*}{ P/E } & $\mathrm{NCHS}$ & 22 & 12,6 & 106 & 61,0 & 46 & 26,4 \\
\hline & CDC & 17 & 9,8 & 120 & 68,9 & 37 & 21,3 \\
\hline & WHO & 21 & 12,0 & 112 & 64,4 & 41 & 23,6 \\
\hline \multirow[t]{3}{*}{ IMC/I } & $C D C$ & 18 & 10,3 & 117 & 67,2 & 39 & 22,5 \\
\hline & WHO & 12 & 6,9 & 116 & 66,7 & 46 & 26,4 \\
\hline & Total $(\mathrm{n}=350)$ & & & & & & \\
\hline \multirow[t]{3}{*}{$\mathrm{P} / \mathrm{l}$} & $\mathrm{NCHS}$ & 29 & 8,3 & 259 & 74,0 & 62 & 17,7 \\
\hline & CDC & 46 & 13,1 & 218 & 62,3 & 86 & 24,6 \\
\hline & WHO & 27 & 7,7 & 231 & 66,0 & 92 & 26,3 \\
\hline \multirow[t]{3}{*}{ P/E } & NCHS & 50 & 14,3 & 210 & 60,0 & 90 & 25,8 \\
\hline & $C D C$ & 41 & 11,7 & 242 & 69,1 & 67 & 19,2 \\
\hline & WHO & 38 & 10,8 & 222 & 63,5 & 90 & 25,8 \\
\hline \multirow[t]{2}{*}{ IMC/I } & CDC & 44 & 12,6 & 232 & 66,3 & 74 & 21,1 \\
\hline & WHO & 25 & 7,1 & 233 & 66,6 & 92 & 26,3 \\
\hline
\end{tabular}

P/l: índice de peso/idade; P/E: índice de peso para estatura; IMC/l: índice de massa corporal para idade; NCHS: National Center for Health Statistics; CDC: Center for Disease Control and Prevention; WHO: World Health Organization.

Tabela 2. Estado nutricional infantil pelo índice E/l, segundo as referências antropométricas e sexo de pré-escolares do município de Viçosa (MG), 2008.

\begin{tabular}{|c|c|c|c|c|c|}
\hline \multirow{2}{*}{ Sexo } & \multirow{2}{*}{ Referência } & \multicolumn{2}{|c|}{ Estatura inadequada } & \multicolumn{2}{|c|}{ Estatura adequada } \\
\hline & & $n$ & $\%$ & $n$ & $\%$ \\
\hline \multirow[t]{3}{*}{ Masculino ( $n=176)$} & $\mathrm{NCHS}$ & 20 & 11,3 & 156 & 88,7 \\
\hline & CDC & 21 & 12,0 & 155 & 88,0 \\
\hline & WHO & 25 & 14,2 & 151 & 85,8 \\
\hline \multirow[t]{3}{*}{ Feminino $(n=174)$} & $\mathrm{NCHS}$ & 21 & 12,0 & 153 & 88,0 \\
\hline & CDC & 22 & 12,6 & 152 & 87,4 \\
\hline & WHO & 26 & 15,0 & 148 & 85,0 \\
\hline \multirow[t]{3}{*}{ Total $(n=350)$} & $\mathrm{NCHS}$ & 45 & 13,0 & 305 & 87,0 \\
\hline & CDC & 48 & 14,0 & 302 & 86,0 \\
\hline & WHO & 58 & 16,5 & 292 & 83,5 \\
\hline
\end{tabular}

E/l: índice de estatura para idade. 
570 | N. SPERANDIO et al.

Considerando a WHO/2006 (atual recomendação do Ministério da Saúde) e o NCHS/ 1977 (recomendação anterior do Ministério da Saúde), ambos como padrão de referência (Tabelas 4 e 5), os valores de sensibilidade, especificidade, valores preditivos positivos e negativos foram mais baixos ao utilizar o CDC, quando comparados com as curvas do NCHS e da $\mathrm{WHO}$, que apresentaram valores mais próximos.

Tabela 3. Coeficiente de Kappa (k) entre os critérios de classificação do estado nutricional, segundo sexo de pré-escolares do município de Viçosa (MG), 2008

\begin{tabular}{|c|c|c|c|c|c|c|c|c|}
\hline \multirow{2}{*}{ Sexo } & \multirow{2}{*}{ Referência } & \multicolumn{2}{|c|}{$\mathrm{P} / \mathrm{l}$} & \multicolumn{2}{|c|}{$\mathrm{P} / \mathrm{E}$} & \multicolumn{2}{|c|}{$\mathrm{IMC/I}$} & \multirow{2}{*}{$\frac{E / l}{E I \times E U T}$} \\
\hline & & $\mathrm{PI} \times \mathrm{EUT}$ & EXP $\times$ EUT & $\mathrm{PI} \times \mathrm{EUT}$ & EXP $\times$ EUT & $\mathrm{PI} \times \mathrm{EUT}$ & EXP $\times$ EUT & \\
\hline \multirow[t]{3}{*}{ Masculino } & CDC e NCHS & $0,40^{\mathrm{a}}$ & $0,66^{a}$ & $0,40^{\mathrm{a}}$ & $0,52^{\mathrm{a}}$ & * & * & $0,80^{\mathrm{a}}$ \\
\hline & NCHS e WHO & $0,88^{a}$ & $0,65^{a}$ & $0,88^{a}$ & $0,62^{a}$ & $*$ & * & $0,91^{\mathrm{a}}$ \\
\hline & CDC e WHO & $0,31^{\mathrm{a}}$ & $0,64^{a}$ & $0,35^{a}$ & $0,67^{\mathrm{a}}$ & $0,56^{\mathrm{a}}$ & $0,80^{a}$ & $0,80^{a}$ \\
\hline \multirow[t]{3}{*}{ Feminino } & CDC e NCHS & $0,16^{\mathbf{b}}$ & $0,56^{\mathrm{a}}$ & $0,34^{\mathrm{a}}$ & $0,53^{\mathrm{a}}$ & * & * & $0,78^{\mathrm{a}}$ \\
\hline & NCHS e WHO & $0,87^{a}$ & $0,80^{\mathrm{a}}$ & $0,86^{\mathrm{a}}$ & $0,85^{a}$ & * & * & $0,89^{a}$ \\
\hline & CDC e WHO & $0,18^{b}$ & $0,52^{a}$ & $0,21^{\mathrm{a}}$ & $0,53^{a}$ & $0,77^{a}$ & $0,82^{a}$ & $0,68^{a}$ \\
\hline \multirow[t]{3}{*}{ Total } & CDC e NCHS & $0,30^{\mathrm{a}}$ & $0,61^{a}$ & $0,31^{\mathrm{a}}$ & $0,52^{\mathrm{a}}$ & * & * & $0,78^{\mathrm{a}}$ \\
\hline & NCHS e WHO & $0,87^{a}$ & $0,71^{\mathrm{a}}$ & $0,80^{\mathrm{a}}$ & $0,88^{a}$ & * & * & $0,87^{a}$ \\
\hline & CDC e WHO & $0,21^{b}$ & $0,52^{a}$ & $0,65^{\mathbf{b}}$ & $0,80^{a}$ & $0,30^{\mathbf{b}}$ & $0,52^{\mathrm{a}}$ & $0,74^{\mathrm{a}}$ \\
\hline
\end{tabular}

${ }^{a} p<0,05 ;{ }^{b} p>0,05 ;{ }^{*}$ Referência do NCHS não possui índice IMC/I.

IMC/I: índice de massa corporal/idade; PI: peso inadequado; EUT: eutrofia; EXP: excesso de peso; El: estatura inadequada; NCHS: National Center for Health Statistics; CDC: Center for Disease Control and Prevention; WHO: World Health Organization.

Tabela 4. Sensibilidade, especificidade, valor preditivo positivo, valor preditivo negativo, considerando a WHO/2006 como "padrão de referência" para classificação do estado nutricional de pré-escolares de Viçosa (MG), 2008.

\begin{tabular}{|c|c|c|c|c|c|c|c|c|c|}
\hline \multirow{2}{*}{ Índice } & \multirow{2}{*}{ Sexo } & \multicolumn{2}{|c|}{ Sensibilidade (\%) } & \multicolumn{2}{|c|}{ Especialidade (\%) } & \multicolumn{2}{|c|}{$\operatorname{VPP}(\%)^{*}$} & \multicolumn{2}{|c|}{ VPN $(\%)^{*}$} \\
\hline & & $\mathrm{NCHS}$ & CDC & $\mathrm{NCHS}$ & CDC & $\mathrm{NCHS}$ & CDC & $\mathrm{NCHS}$ & CDC \\
\hline & Masculino & & & & & & & & \\
\hline \multirow[t]{2}{*}{$P / I$} & Peso inadequado & 99 & 55 & 97 & 87 & 81 & 30 & 99 & 95 \\
\hline & Excesso de peso & 85 & 75 & 98 & 89 & 96 & 70 & 94 & 84 \\
\hline \multirow[t]{2}{*}{$\mathrm{P} / \mathrm{E}$} & Peso inadequado & 99 & 62 & 92 & 88 & 76 & 50 & 98 & 94 \\
\hline & Excesso de peso & 89 & 54 & 98 & 94 & 97 & 66 & 94 & 80 \\
\hline \multirow[t]{2}{*}{ IMC/I } & Peso inadequado & $* *$ & 92 & $* *$ & 87 & $* *$ & 50 & $* *$ & 98 \\
\hline & Excesso de peso & ** & 74 & ** & 98 & ** & 98 & $* *$ & 89 \\
\hline \multirow[t]{2}{*}{$E / l$} & Estatura inadequada & 86 & 74 & 99 & 98 & 97 & 95 & 97 & 94 \\
\hline & Feminino & & & & & & & & \\
\hline \multirow[t]{2}{*}{$P / l$} & Peso inadequado & 85 & 30 & 98 & 80 & 92 & 16 & 98 & 90 \\
\hline & Excesso de peso & 77 & 65 & 98 & 86 & 94 & 68 & 91 & 84 \\
\hline \multirow[t]{2}{*}{$\mathrm{P} / \mathrm{E}$} & Peso inadequado & 95 & 30 & 96 & 81 & 83 & 35 & 98 & 86 \\
\hline & Excesso de peso & 95 & 65 & 93 & 82 & 84 & 70 & 97 & 85 \\
\hline \multirow[t]{2}{*}{ IMC/I } & Peso inadequado & $* *$ & 98 & ** & 90 & ** & 66 & ** & 99 \\
\hline & Excesso de peso & ** & 80 & ** & 98 & ** & 94 & $* *$ & 92 \\
\hline$E / l$ & Estatura inadequada & 77 & 65 & 98 & 97 & 88 & 85 & 94 & 92 \\
\hline
\end{tabular}

*VPP: valor preditivo positivo; VPN: valor preditivo negativo; ${ }^{* *}$ Referência do NCHS não possui índice IMC/I.

IMC/I: índice de massa corporal/idade; NCHS: National Center for Health Statistics; CDC: Center for Disease Control and Prevention. 
Tabela 5. Sensibilidade, especificidade, valor preditivo positivo, valor preditivo negativo, considerando a NCHS/1977 como "padrão de referência" para classificação do estado nutricional de pré-escolares de Viçosa (MG), 2008.

\begin{tabular}{|c|c|c|c|c|c|c|c|c|c|}
\hline \multirow{2}{*}{ Índice } & \multirow{2}{*}{ Sexo } & \multicolumn{2}{|c|}{ Sensibilidade (\%) } & \multicolumn{2}{|c|}{ Especialidade (\%) } & \multicolumn{2}{|c|}{$\operatorname{VPP}(\%)^{*}$} & \multicolumn{2}{|c|}{ VPN $(\%)^{*}$} \\
\hline & & CDC & WHO & CDC & WHO & $C D C$ & WHO & CDC & $\mathrm{WHO}$ \\
\hline & Masculino & & & & & & & & \\
\hline \multirow[t]{2}{*}{$\mathrm{P} / \mathrm{I}$} & Peso inadequado & 60 & 82 & 88 & 98 & 40 & 99 & 94 & 96 \\
\hline & Excesso de peso & 96 & 99 & 86 & 81 & 60 & 71 & 98 & 99 \\
\hline \multirow[t]{2}{*}{ P/E } & Peso inadequado & 45 & 76 & 87 & 99 & 48 & 99 & 86 & 92 \\
\hline & Excesso de peso & 56 & 99 & 92 & 95 & 77 & 90 & 82 & 99 \\
\hline \multirow[t]{2}{*}{ E/l } & Estatura inadequada & 77 & 99 & 98 & 97 & 87 & 89 & 96 & 99 \\
\hline & Feminino & & & & & & & & \\
\hline \multirow[t]{2}{*}{$\mathrm{P} / \mathrm{I}$} & Peso inadequado & 42 & 95 & 82 & 93 & 30 & 89 & 92 & 86 \\
\hline & Excesso de peso & 75 & 94 & 86 & 89 & 64 & 75 & 91 & 98 \\
\hline \multirow[t]{2}{*}{ P/E } & Peso inadequado & 40 & 83 & 90 & 99 & 45 & 95 & 84 & 96 \\
\hline & Excesso de peso & 60 & 85 & 91 & 98 & 75 & 95 & 82 & 93 \\
\hline E/l & Estatura inadequada & 81 & 99 & 96 & 94 & 80 & 98 & 95 & 99 \\
\hline
\end{tabular}

*VPP: valor preditivo positivo; VPN: valor preditivo negativo.

CDC: Center for Disease Control and Prevention; WHO: World Health Organization.

\section{I S C U S S Ã O}

Apesar de a desnutrição continuar sendo um dos problemas de saúde pública que mais acometem a população infantil, nas últimas décadas verifica-se o processo de transição nutricional, que tem sido apontado como fator fundamental para aumento do número de casos de sobrepeso e obesidade ${ }^{17,18}$. Modificações do hábito alimentar associadas a alterações demográficas, socioeconômicas e epidemiológicas mostram redução progressiva da desnutrição e aumento da obesidade ${ }^{18,19}$.

O excesso de peso predispõe as crianças a várias complicações, como problemas respiratórios, diabetes mellitus, hipertensão arterial dentre outros ${ }^{18,19}$. A detecção do excesso de peso na infância é importante, pois permite intervenções precoces e prevenção de complicações. Quanto mais tempo esse quadro se mantiver, mais difícil será o tratamento e a incorporação de novos hábitos $^{19}$.

Torres et al. ${ }^{20}$, ao avaliarem crianças de 0 a 60 meses, utilizando as curvas de crescimento do CDC/2000 e da WHO/2006, também encontraram maior prevalência de baixo peso, segundo índice P/I, adotando a proposta do CDC/2000; porém, para o índice $\mathrm{P} / \mathrm{E}$, a maior prevalência de baixo peso foi detectada pela WHO/2006. Já Orellana et al. ${ }^{21}$, em estudo comparativo utilizando as curvas do NCHS/1977 e da WHO/2006 para avaliar estado nutricional de crianças de 0 a 59 meses, encontraram que, para o índice P/l, a prevalência de baixo peso foi maior ao adotar o NCHS/1977. Porém, as diferenças não foram significantes.

Stahelin", ao avaliar crianças com menos de 60 meses em uma creche de Florianópolis e comparar a referência do CDC/2000 com a WHO/ 2006, observou que, quando a avaliação foi feita utilizando a WHO/2006, foram detectados maiores valores de sobrepeso. Resultado semelhante ao encontrado no presente estudo: a proposta da WHO apresentou os maiores valores de excesso de peso.

Confrontando as curvas do CDC/2000 com as da WHO/2006, temos que a média de peso das crianças acima de oito meses, na referência do CDC/2000, é maior, sendo, consequentemente, maior a prevalência de baixo peso, enquanto a referência da WHO/2006 apresentará maiores prevalências de excesso de peso ${ }^{9,10}$.

Segundo De Onis et al. ${ }^{22}$, o novo padrão WHO/2006, baseado em crianças amamentadas, 
certamente identificará de maneira mais precoce crianças com sobrepeso e obesidade: uma epidemia crescente em todo mundo. As curvas do NCHS/1977 e CDC/2000 são criticadas por serem baseadas em amostras com uma prevalência substancial de sobrepeso, o que faz com que os percentis superiores sejam superestimados, deixando assim de identificar muitas crianças com excesso de peso. Conclusões errôneas eram obtidas ao se compararem crianças em aleitamento materno com crianças em aleitamento artificial, o que contribuía para introdução precoce de alimentação complementar devido à identificação incorreta de crescimento lento nas crianças em aleitamento materno.

Com relação ao índice E/I, outros estudos também encontram maior prevalência de deficit de estatura ao utilizar a WHO/2006. Damaceno et al..$^{23}$, ao avaliarem o perfil nutricional de crianças de 6 a 24 meses de idade, encontraram uma prevalência de deficit estatural de 2,1\%, 2,1\% e $4,2 \%$ ao utilizarem as três curvas de crescimento infantil: NCHS, CDC e WHO, respectivamente. Outro estudo que comparou as curvas do NCHS e da WHO mostrou que os percentis de deficit de E/l foram em média 10,0\% maiores com a curva da WHO, na avaliação de crianças com menos de 60 meses $^{24}$.

Silveira et al. ${ }^{25}$, ao compararam o estado nutricional de crianças com até 24 meses da região do Alto Jequitinhonha (MG) segundo as curvas de crescimento da WHO e do NCHS, encontraram que as curvas da WHO tiveram maior sensibilidade para detecção de deficit peso para altura (wasting), exceto para crianças acima dos 11 meses, e para o deficit altura para idade (stunting).

Esses resultados são compatíveis com os apresentados no Estudo Multicêntrico de Referência para o Crescimento da WHO (MGR: Multicentre Growth Reference Study), que indicou que crianças com baixa estatura tendem a ser diagnosticadas mais precocemente ao utilizar a referência da WHO/2006, em comparação com o NCHS/1977 e o CDC/200026.
Os percentuais de baixa estatura encontrados $(16,5 \%)$ pela curva da WHO/2006 são maiores que os apresentados pela Pesquisa Nacional de Demografia e Saúde (PNDS) ${ }^{27}$, em que a prevalência de deficit estatural em crianças menores de cinco anos foi de $7 \%$. Porém, devido ao ponto de corte -1 escore-Z é mais sensível que 0 -2 escore-Z, adotado pela PNDS, já era esperada uma prevalência maior de deficit estatural.

Diferenças foram observadas entre as curvas utilizadas, com variações dependendo do sexo e do índice antropométrico. Alguns pontos podem ser colocados para explicar tais variações, como, por exemplo, as características de cada referência. A amostra da WHO/2006 foi multiétnica, composta por crianças que foram amamentadas, exclusiva ou predominantemente, até os 4 meses, e envolveu quatro continentes, enquanto as amostras do NCHS/1977 e CDC/2000 foram compostas por crianças americanas alimentadas por fórmulas infantis ${ }^{8,21}$. Essas variações com relação às metodologias contribuem para as diferenças encontradas com relação às prevalências tanto de deficits como de excesso de peso.

A curva de crescimento do CDC/2000 não apresentou boa concordância $(k)$ com as demais; já a WHO/2006 e o NCHS/1977 apresentaram boa concordância para ambos os sexos. Além disso, observam-se valores semelhantes de sensibilidade e valor preditivo positivo entre a WHO/2006 e o NCHS/1977, mesmo agrupando os sexos, reforçando a boa concordância ( $k$ ) encontrada. Logo, a substituição da curva do NCHS pela WHO, considerando as condições e os critérios adotados pelo presente estudo, não implicaria diferença significante com relação aos diagnósticos obtidos, porém algumas crianças diagnosticadas por meio de uma curva podem não receber o mesmo diagnóstico quando utilizada outra curva, o que reforça a importância de se trabalhar com outros indicadores para avaliação do estado nutricional infantil, além da antropometria. 


\section{CONCLUSÃO}

A WHO/2006 apresentou maiores valores de sensibilidade principalmente para estatura inadequada. Logo, a adoção desse padrão antropométrico pode tornar precoce a identificação de deficits nutricionais, possibilitando assim intervenções que possam corrigir essas distrofias e permitir um desenvolvimento adequado. A adoção das curvas propostas pela WHO pelos profissionais da área da saúde permite uma avaliação mais adequada do crescimento infantil por tratar-se de um padrão de crescimento atual, recomendado para uso internacional. No caso de crianças brasileiras e no impacto sobre os inquéritos nutricionais e estudos de prevalência, a boa concordância do NCHS e da WHO acaba por gerar resultados semelhantes. Em avaliações individuais, recomenda-se, a exemplo do preconizado para as curvas do NCHS e CDC, que a avaliação antropométrica seja analisada em conjunto com outros indicadores do estado nutricional infantil.

\section{A GRADECIMENTOS}

Ao Conselho Nacional de Desenvolvimento Científico e Tecnológico (CNPq) o apoio financeiro, aos responsáveis pelos pré-escolares, às crianças estudadas, aos funcionários das instituições e aos estagiários voluntários de nutrição da Universidade Federal de Viçosa a contribuição durante a coleta dos dados.

\section{COLABORADORES}

N. SPERANDIO foi responsável pela coleta e análise dos dados antropométricos, levantamento bibliográfico para elaboração deste artigo, pela escrita e formatação do artigo. L.F.R. SANT'ANA e S.C.C. FRANCESCHINI colaboraram na elaboração e revisão do artigo. S.E. PRIORE foi responsável pela orientação durante toda coleta dos dados, além de contribuir para elaboração e revisão do artigo.

\section{REFERÊ N CIAS}

1. Silva HGV, Chiara VL, Barros ME, Rego AL, Ferreira A, Pitasi BA, et al. Diagnóstico do estado nutricional de escolares: comparação entre critério nacional e internacional. J Pediatr. 2008; 84(6):550-5. doi: 10.1 590/S0103-05822009000200004.

2. Sigulem DM, Devincenz UM, Lessa AC. Diagnóstico do estado nutricional da criança e do adolescente. J Pediatr. 2000; 76(3):275-84. doi: 10.1590/S010 3-05822009000200004.

3. Ferreira HS. Avaliação nutricional de crianças pelo método antropométrico. In: Ferreira HS. Desnutrição: magnitude, significado social e possibilidade de prevenção. Maceió: EDUFAL; 2000. v.1.

4. Monte CMG, Muniz HF, Dantas Filho S. Crescimento e desenvolvimento da criança menor de cinco anos. In: Monte CMG. Promoção da nutrição das crianças menores de 5 anos no dia-a-dia da comunidade. Vitória: UFES; 2001. p.106-139.

5. Zeferino $A M B$, Filho $A A B$, Bettiol $H$, Barbiere MA. Acompanhamento do crescimento. J Pediatr. 2003; 79(1):23-32. doi: 10.1590/S0103-0582200900020 0004.

6. Corso ACT, Botelho L. Avaliação do estado nutricional de crianças menores de seis anos de idade do município de Florianópolis [mestrado]. Santa Catarina: Universidade Federal de Santa Catarina; 2000.

7. Post CLA, Victora CG, Barros AJD. Entendendo a baixa prevalência de deficit de peso para estatura em crianças brasileiras de baixo nível sócioeconômico: correlação entre índices antropométricos. Cad Saúde Pública. 2000; 16(1):73-82. doi: 10.1590/S0102-311X2000000100008.

8. Soares NT. Um novo referencial antropométrico de crescimento: significados e implicações. Rev Nutr. 2003; 16(1):93-103. doi: 10.1590/S1415-527320 03000100010.

9. Stahelin L. Avaliação do estado nutricional das crianças menores de cinco anos em uma creche no município de Florianópolis segundo a curva de referência da OMS 2006 e comparação do diagnóstico nutricional com a curva de referência do CDC 2000 [mestrado]. Santa Catarina: Universidade Federal de Santa Catarina; 2007.

10. Filho MB, Souza Al, Miglioli TC, Santos MC. Anemia e obesidade: um paradoxo da transição nutricional brasileira. Cad Saúde Pública. 2008; 24(2):247-57. doi: 10.1590/S1415-52732003000100010.

11. Schoeps DO. Crescimento e estado nutricional de pré-escolares de creches filantrópicas de Santo André: a transição epidemiológica nutricional do Município [mestrado]. São Paulo: Universidade de São Paulo; 2004.

12. Sotelo YOM, Colugnati FAB, Taddei JAAC. Prevalência de sobrepeso e obesidade entre escolares da rede pública segundo três critérios de diagnós- 
574 | N. SPERANDIO et al.

tico antropométrico. Cad Saúde Pública. 2004: 20(1):233-40. doi: 10.1590/S0102-311X200400 0100040 .

13. World Health Organization. Working Group on Infant Growth. An evaluation of infant growth: the use and interpretation of anthropometry in infants. Bull WHO. 1995; 73(2):165-74.

14. Fox E, Kuo J, Tilling L, Ulrich C. User's manual: sigma stat: statistical software for windows. Jandel, Germany, 1994.

15. Fleiss JL, Cohen J, Everitt BS. Large sample standard errors of kappa and weighted kappa. Psychol Bull. 1969; 12(1):323-7.

16. Landis JR, Koch GG. The measurement of observer agreement for categorical data. Biometrics. 1977; 33(1):159-74. doi: 47075978.

17. Menezes RC, Egito, OM. Consumo energético-proteico e estado nutricional de crianças menores de cinco anos, no estado de Pernambuco, Brasil. Rev Nutr. 2007; 20(4):337-47. doi: 10.1590/S1415-52 732007000400001

18. Francischi RPP, Pereira LP, Freitas CS, Klopfer MS, Santos RS, Vieira P, et al. Obesidade: atualização sobre etiologia, morbidade e tratamento. Rev Nutr. 2000; 13(1):17-28. doi: 10.1590/S1415-52732007 000400001.

19. Batista FM, Rissin A. A transição nutricional no Brasil: tendências regionais e temporais. Cad Saúde Pública. 2003; 19(1):181-91. doi: 10.1590/S0102-3 $11 \times 2003000700019$

20. Torres $A A L$, Furumoto RAV, Alves ED. Avaliação antropométrica de pré-escolares - comparação entre os referenciais: NCHS 2000 e OMS 2005. Rev Eletr Enfe [Internet]. 2007 [acesso 2009 dez 15]; 9(1):166-75. Disponível em: <http://www.fen. ufg.br/revista/v9/n1/v9n1a13.htm>. doi: 10.5216/ ree.v9i1.6710.

21. Orellana JDY, Santos RV, Coimbra Jr CEA, Leite MS. Avaliação antropométrica de crianças indígenas menores de 60 meses, a partir do uso comparativo das curvas de crescimento NCHS/1977 e OMS/2005. J Pediatr. 2009; 85(2):117-21. doi: 10.1590/S00 21-75572009000200006.

22. De Onis M, Onyango AW, Borghi E, Garza C, Yang $\mathrm{H}$. Comparison of WHO Child Growth Standards and National Centre for Health Statistics/ WHO international growth standards: implication for child health programmes. Public Health Nutr. 2006; 9(1):942-7. doi: 10.1017/PHN20062005.

23. Damaceno RJP, Martins PA, Devincenzi MU. Estado nutricional de crianças atendidas na rede pública de saúde do município de Santos, São Paulo, Brasil. Rev Paul Pediatr. 2009; 27(2):139-47. doi: 10.1590/S 0103-05822009000200004.

24. De Onis M, Onyango AW, Borghi E, Garza C, Yang $\mathrm{H}$; WHO Multicentre Growth Reference Study Group. Comparison of the World Health Organization (WHO) Child Growth Standards and the National Center for Health Statistics/WHO international growth reference: implications for child health programmes. Public Health Nutr. 2006; 9(7):942-7.

25. Silveira FJF, Lamounier JA. Avaliação nutricional de crianças do Vale do Alto Jequitinhonha com a utilização das novas curvas de crescimento do NCHS e da OMS. Rev Paul Pediatr. 2009; 27(2):133-8. doi: 10.1590/S0103-05822009000200003.

26. De Onis M, Garza C, Onyango AW, Borghi E. Comparison of the WHO Child Growth Standards and the CDC 2000 Growth Charts. J Nutr. 2007; 137(1):144-8.

27. Brasil. Ministério da Saúde. PNDS-2006: pesquisa nacional de demografia e saúde da criança e da mulher [Relatório]. Brasília: Ministério da Saúde; 2008.

Recebido em: 5/5/2010

Versão final reapresentada em: 9/11/2010 Aprovado em: 16/12/2010 